\title{
Optical Based Sensor Prototype for Continuous Monitoring of the Blood Pressure
}

\author{
Zachary Cohen and Shyqyri Haxha, Senior Member, IEEE.
}

\begin{abstract}
In this paper, we report a prototype ring sensor device for continuous measurement of blood pressure with the use of our, previously developed, heart rate monitoring ring device. An experiment is described where the heart rate device provides the voltage output of the heart using the transmission photoplethysmography (PPG) method and predicts the blood pressure's value to $\pm 5 \%$ of its true value. We report a novel potential non-invasive, low cost, continuous heart rate and blood pressure monitoring device that uses transmission PPG instead of the traditional cuff method to observe the changes in volume of the pressure through the arteries of the finger. The continuous samples are averaged out constantly. We employed the PPG technique to optically determine the blood volume changes in the arteries of the finger. A Pearson's product moment correlation coefficient proved an $r$ value of 0.86 showing strong linear correlation between the average voltage of the heart rate and the corresponding blood pressure. The proposed blood pressure ring sensor device was tested and benchmarked (against Nonin 2120 benchmark blood pressure device) four participants for a continuous period of four hours, where the average Mean Arterial Pressure (MAP) (using Nonin 2120) for four hours was at $98.92 \mathrm{mmHg}$ and the average predicted MAP was at $92.8 \mathrm{mmHg}$, which demonstrates an accuracy of $93.8 \%$.The average real systolic pressure (using Nonin 2120) was at $144.25 \mathrm{mmHg}$ and the predicted average systolic pressure was at $132.77 \mathrm{mmHg}$ which shows an accuracy of $92 \%$. The average real diastolic pressure (using Nonin 2120) was at $76.25 \mathrm{mmHg}$ and the predicted diastolic pressure was $72.7 \mathrm{mmHg}$, showing an accuracy of $95.5 \%$.
\end{abstract}

Index Terms- Optical sensors, Wearable sensors, Medical services.

\section{INTRODUCTION}

ADVANCED sensor technology has progressed significantly during the last decade for personal healthcare monitoring (PHM), for outpatients at home and patients whilst admitted to hospital. It is highly important for any developed PHM sensor technology to be comfortable, accurate and low cost. Such developed sensor technology should be able to monitor patients and non-patients health key vitals at the home on a day to day basis, which will allow the medical personnel to use this technology remotely at a very low cost to monitor health status. Cardiovascular diseases (CDVs) are the heart disorders including heart attacks and strokes. Out of 17.5 million deaths each year from CDVs, $80 \%$ are due to heart attacks and strokes [1] and it is predicted to increase to 24.2 million deaths by 2030 due to poor healthcare and lack of physical exercise [2]. According to the British heart foundation, seven out of ten people survive heart attacks [3]. If a sensor device could predict the patient's heart routine before the heart attack with the aid of a continuous heart rate monitor, it may be possible to anticipate an oncoming heart attack before it actually happens.

Many healthcare devices can be used at home to measure (inter alia) heart rate, oxygen saturation, glucose levels, body temperature and blood pressure [4-11]. A traditional method of measuring blood pressure is by using a sphygmomanometer [12]. These devices are usually used on the upper arm (concerning the brachial artery [13]), where the blood pressure can be deciphered by a user listening to the blood in the artery after being cut off. A standard reading for blood pressure is $120 / 80$ [14]. The numerator is the systolic pressure, when the heart contracts and pushes the blood through the arteries. The denominator is the diastolic pressure, whilst the heart is relaxing and refilling with blood. Thus the blood pressure could be seen as a range, as it is constantly changing with every heartbeat. Wearable sensors are becoming highly desirable within the sensor technologies that people use every day. Many 'smart' watches use the wearable sensors to provide the user more information about their health vitals. Many sensors can be incorporated and integrated with flexible materials to gather more information such as body temperature, heart rate, oxygen saturation level and glucose [15-25]. Recently the authors in [23] have demonstrated a ring sensor for determining and monitoring the oxygen saturation level in the human blood.

Currently the blood pressure is rarely monitored on a continuous basis throughout the day. It is mainly measured when a patient visits the surgery. Users have not felt the need to monitor their blood pressure in a similar way to the manner in which they have begun to monitor their heart rate, and for many years they have been taking their own temperature when feeling unwell. Unfortunately, this has led to a stagnation of innovation with the sphygmomanometer cuff method being the favoured but old fashioned technique. This might be worth reconsidering as users seek ever more accurate and complete information about their own personal health.

A new variation of the cuff method is a blood pressure dock, produced by iHealth [27]. A user's iPhone is plugged into an iHealth dock where a cuff is attached to it. The measurement is taken and sent to the phone's health app and costs around $£ 70$ $(\$ 100)$. The battery used is a $3.7 \mathrm{~V} \mathrm{Li}$ ion, the documentation for the device doesn't state battery life, but considering that it is a lower voltage than the operational voltage of $5 \mathrm{~V}$ of the device, 
it is unlikely to be more than a couple of hours long. This method can also be produced as a wireless wrist strap, but still uses the uncomfortable inflatable cuff, this costs $£ 55$ (\$80) [28]. The only other apps concerning blood pressure have to be imported into the app by the user. The proposed ring sensor in this study will set out a way to continuously measure blood pressure without using the uncomfortable cuff method. A device has been designed by Massachusetts Institute of Technology (MIT) which uses a method called pulse wave velocity (PWV) [29]. PWV measures two points along the artery and utilises Newton's second law of motion;

$$
F=m a
$$

Where $F$ is the force, $m$ is the mass and a, the acceleration of the blood. The two points of the artery will be used to deduce the acceleration within the formula; $a=((v-u) / t)$ where $v$ is the final velocity, $u$, the initial velocity and $t$ is the time. By using the formula; $P=F / A$, where $P$ is pressure, $F$ is force and $A$ is the cross sectional area of the artery, we can obtain the equation:

$$
P=\frac{(m a)}{A}
$$

This will figure out the pressure of the arterial blood through the finger. The main issue with the proposed PWV method [29] is that many assumptions must be made including that the area will always be the same. Assuming the device will be calibrated to the specific person of area of the known artery, this will not enable the user to use the measurement on a different finger as the vessels and capillaries will be different throughout the body. If the method is used for a person still growing, the area will gradually change as their body gets bigger and thus will decalibrate the individual.

Our proposed blood pressure ring sensor will not use the cuff method or the pulse wave velocity method $[14,29]$ and it will cause no discomfort to the user. In our proposed sensor we use the photoplethysmography method (PPG) to implement a heart rate monitor with low cost and effective (simple, not complex) components. The PPG is a well-established method that optically determine the changes in the volume. It should be mentioned that in this research paper we are using existing conventional PPG method to measure and monitor the blood pressure in real-time via a very affordable heart rate monitor. It uses each individuals' pre calibrated values to predict the blood pressure with up to $90 \%$ accuracy with use of the product moment coefficient. To the best of our knowledge a blood pressure monitor with a heart rate and a pre made oxygen saturation monitor is not reported in the literature, and it does not exist in the market currently.

\section{RESULTS AND DISCUSSIONS-HEART RATE MONITORING IMPLEMENTATION}

The proposed device uses an LDR and an LED to observe the pulse, where the microcontroller will read the voltage output of the resistance from the LDR. The LDR and LED are placed within an elastic material for the comfort of the user, stimulate a greater pulse and allow the LDR to ameliorate the effect of any ambient light.

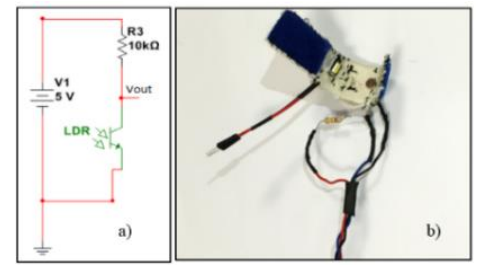

Fig. 1. a) Circuit design for the LDR. b) Real implementation of LDR circuit with LED enclosed in the elastic material held to the finger by Velcro.

When the circuit output is seen on an oscilloscope, there is a visible rise in voltage as the heart beats. As there is more blood within the capillaries of the finger, less light reaches the LDR. As less light reaches the LDR, a higher resistance is recorded by the LDR. As voltage is proportional to resistance in Ohm's law, a higher voltage is achieved. The heart rate signal is filtered and then amplified, in order to be seen in the oscilloscope at an appropriate level, for the microcontroller to be able to read it. The heart rate device can be seen in figure 3 . When the signal is received, there is a large DC offset of $\sim 2 \mathrm{~V}$. This disrupts the analogue reading and becomes negligible once amplified. The DC offset must be removed prior to amplification by filtering the input signal. A suitable heart rate monitor should be able to read a heart rate across a high range. The chosen range of this heart rate monitor is from 60 beats per minute (BPM) to 200BPM. A band-pass filter can be implemented to filter out any other unwanted frequencies and remove the unwanted DC offset. This is made up of a High Pass and a Low Pass filter. The second order band-pass filtering design is completed with a high-pass filter of $10 \mathrm{~K} \Omega$ resistance. A $14.7 \mu \mathrm{F}$ capacitor is used to cut off $1.08 \mathrm{~Hz}$ (65BMP) and a low-pass filter with resistance $10 \mathrm{~K} \Omega$ and capacitance of $4.7 \mu \mathrm{F}$ is used to cut off $3.38 \mathrm{~Hz}$ (203BPM), giving an overall centre frequency:

$$
f r=\sqrt{f l \times f h}
$$

This makes the centre frequency equal to $1.91 \mathrm{~Hz}$. The overall high pass to low pass filter design is shown below in Figure 2.

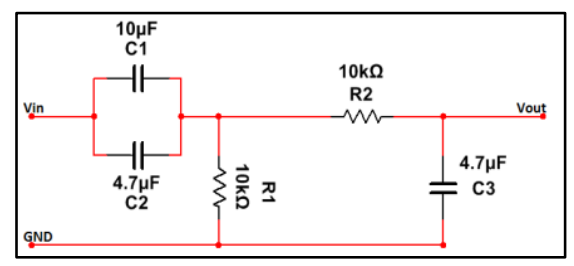

Fig. 2. High pass to low pass filter design.

The oscilloscope shows a signal of $0.2 \mathrm{~V}$ output when the heartbeat is made. A resistor and capacitor has been added again 
in order to smooth the output and diminish the DC off-set produced by a LM386 OP-AMP. The circuitry design was implemented on a breadboard which produces high capacity where a printed circuit board (PCB) with components soldered on it has been implemented, as shown in Figure 3.

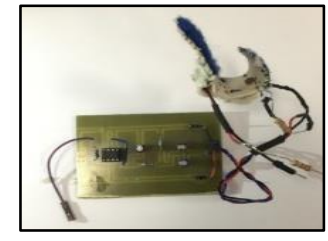

Fig. 3. PCB circuit attached to the optical sensor.

Fig. 3. PCB circuit with LDR ring sensor integrated filters, OPAMP and Vout.

After the heart beat signal has been observed, filtered and amplified, next we performed the microcontroller programming in order to identify the shape of the heartbeat waveform and find the heart rate per minute. An Arduino UNO has been used, as it has the libraries required and it is simply to be programed. The Arduino read the output of the heart rate monitor in an analogue form from 0-1023. As the Arduino's voltage at analogue read of 0 is $0 \mathrm{v}$ and an analogue read of 1023 is $5 \mathrm{~V}$, it is easy to convert the reading into the voltage (Voltage $=$ analogue reading $\times(5 /$ 1023)). A spreadsheet is produced which recorded the output voltage against time (one voltage reading every $50 \mathrm{~ms}$ ).

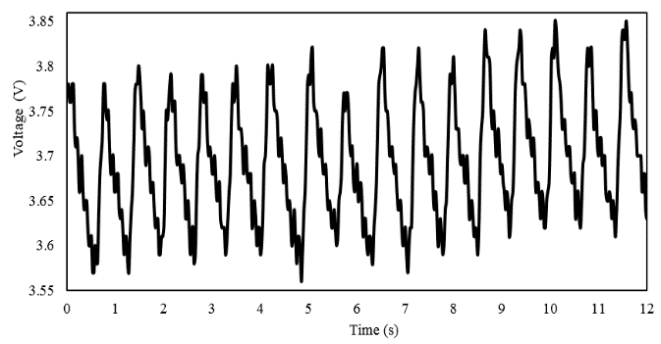

Fig. 4. Output of the LDR sensor as a function of time.

As can be seen from Figure 4, there is a change in voltage as the heart is pumping. Figure 4 shows 17 pulses in 12 seconds $(0.2$ minutes). This would give a heart rate of 85BPM (17/0.2). This heart rate monitor is benchmarked using in-house a Nonin 2120 device that measures heart rate, oxygen saturation and blood pressure and costs around $£ 1500$ [30].

A closer look at the results from one heartbeat, illustrated in Figure 5, can show that the maximum voltage (red line) is at $3.80 \mathrm{~V}$ and the minimum at $3.57 \mathrm{~V}$. This gives an overall voltage of $0.25 \mathrm{~V}$ per beat with the average $\sim 3.69 \mathrm{~V}$. When the reading is above the average $3.7 \mathrm{~V}$, a pulse is present.

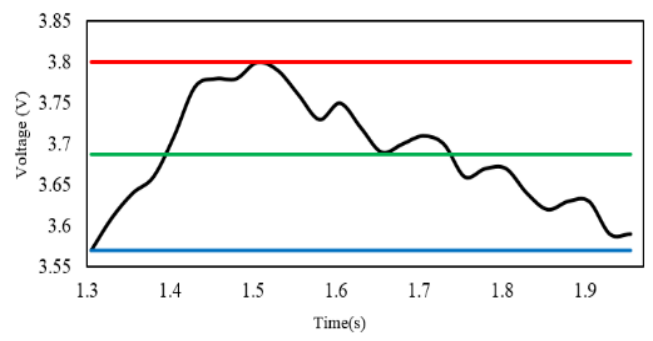

Fig. 5. One heart beat measurement (where the red line is the maximum voltage, blue, the minimum and green, the average).

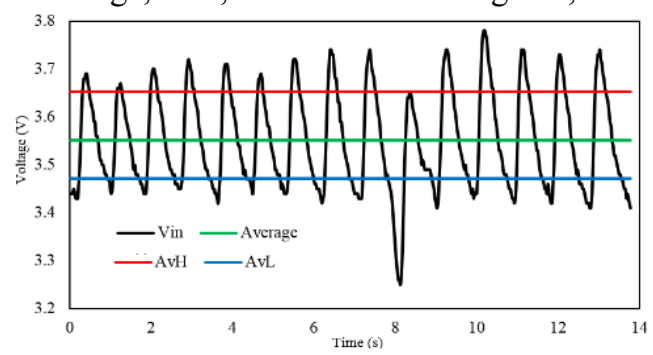

Fig. 6. Another heart beat analysis from the Vin. Showing 15 beats in 14 seconds. A heart rate of 65BPM.

Figure 6, shows another recorded heart beat from Vin. It is clear from this figure that the heart rate of 65BPM derived from the reading of 15 beats in 14 seconds. Whilst this can be manually determined, the Arduino can be programmed to understand the definition of what a 'heart beat' is using only the Vin and time. This can be achieved by finding the average (green line) of $3.55 \mathrm{~V}$. Every time the Vin is above $3.55 \mathrm{~V}$, a counter can take note. There are $\sim 16$ voltage readings above this threshold within every heartbeat. There are a total of 241 readings above the 3.55 threshold within $13.775 \mathrm{~s}$. Taking the total instances of voltages above the threshold by the amount of voltages per heartbeat gives us the amount of beats within the reading; $(241 / 16=15)$. As can be seen from these analyses, it can be confirmed that there are 15 beats within the 13.775 seconds leading to a heartrate of 65BPM $((15 / 13.775) / 60)$. Now it is possible to mathematically calculate the heartrate with the use of Excel. After this, we have performed the microcontroller programming in order to produce a beat against a timer.

The pulse can be programmed by instructing the microcontroller that when the voltage is above the average reading, note a recording. As it is necessary to only note a pulse when the reading is above the average reading once, a counter can be implemented which can state that the value has to go down (below the average) before it can go up again. Now the microcontroller can acknowledge a pulse, a timer can be implemented which will run against the counter. The microcontroller reads 30 pulses before the counter and timer is reset. A liquid crystal display (LCD) has been used to display the results of the heart rate whenever the counter reached the 30 beats. This is due to the possibility of null readings and ensures that faults within the reading can be reset.

The ring heart rate monitor can be placed comfortably on the 
finger. If the heart rate is too high or too low, the microcontroller can send a message via Bluetooth, internet or radio frequency (RF) and so alerting any nominated medical professional(s) to the readings. The circuit has very little technology associated with it and is very affordable, which produces a gateway for medical carers to monitor the patient's status.

\section{RESULTS AND DISCUSSIONS-BLOOD PRESSURE IMPLEMENTATION}

Md. Manirul Islam et al [31] produce a non-invasive continuous blood pressure monitoring device with a use of the photoplethysmography method. In our proposed heart rate monitoring device, we used the similar theoretical method [31] to implement the heart rate monitor. Using a high intensity LED and LDR around the finger, blood pressure can be deduced after calibration by means of using the current blood pressure as the base value. As the light is received through the LDR during systole, the LDR value is at a maximum and as the light is received through the LDR during diastole, the resistance is at a minimum. The resistance of the LDR is proportional to the blood pressure. D. Zeng et al [32] reported a continuous blood pressure monitor that is placed as a ring device. The method involves comparing the user's ECG 'QRS' complex to distinguish the change in volumes of the arterial pressure where this paper reports a method that only uses the heart rate, therefore no ECG nodes would need to connect to other extremities of the body. This method proved successful however the ring requires three dry ECG nodes to be connected to the user for the measurement. This makes the ring less convenient and indiscrete for a user who may need to measure their blood pressure continuously at home [33 - 34]. Taking a view of the heart rate shown in Figure 7 , produced by the proposed heart rate device, it can be seen that the theoretical systolic blood pressure (SBP) can be calculated via the use of the maximum value of the heart rate (red line on Figure 7) and the diastolic blood pressure (DBP) (blue line on Figure 7).

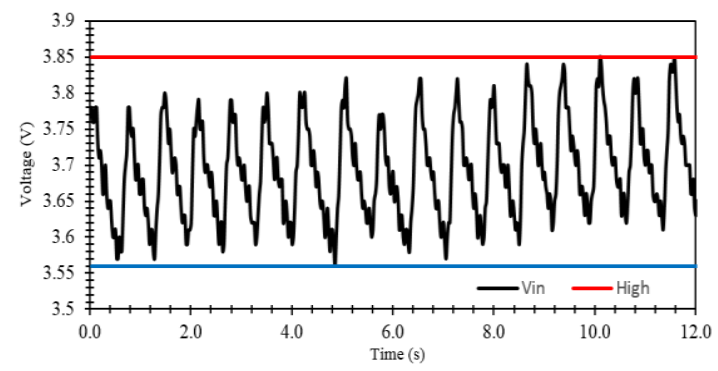

Fig. 7. Heart rate measurement where the wave form consists of 17 heart beats in 12 seconds; 85BPM.

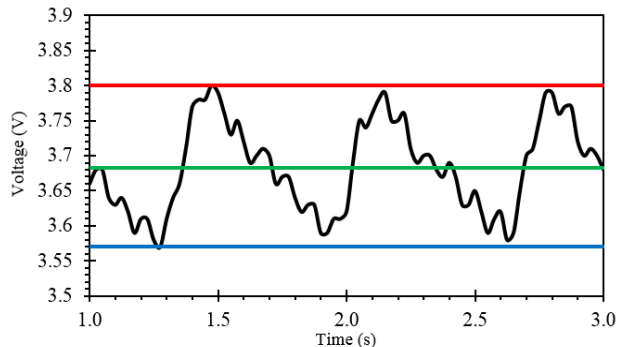

a)

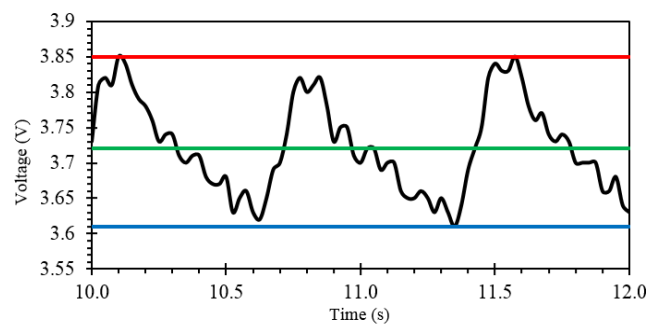

Fig. 8. a) A portion of the heart rate graph from Figure 1 with maximum voltage amplitude of $3.8 \mathrm{~V}$ and minimum of $3.57 \mathrm{~V}$. b) A portion of the figure 1 heart rate graph with maximum voltage of $3.85 \mathrm{~V}$ and a minimum voltage of $3.6 \mathrm{~V}$.

Figure 8 a) and Figure 8 b) illustrate the comparison of two separate heart rates. The heart rate can be easily determined. Both Figures (Fig. 8 a) and (Fig. 8 b) have about three heart beats in two seconds, making a heart rate of $\sim 90$ BPM. Fig. 8 a) has a maximum voltage of $3.8 \mathrm{~V}$ and a minimum of $3.57 \mathrm{~V}$. Fig. $8 \mathrm{~b}$ ) has a maximum voltage of $3.85 \mathrm{~V}$ and a minimum voltage of $3.61 \mathrm{~V}$. As there is a higher voltage within the second, there is a higher resistance and as there is a higher resistance, less light is getting to the LDR compared to the first. As less light is getting to the LDR, there is a higher volume of blood within the finger. Since there is a higher volume of blood, there is a higher blood pressure within Fig. 8 b) with a maximum of $3.85 \mathrm{~V}$. The same implies for the minimum voltage values. As Fig. 8 b) shows a $+0.4 \mathrm{~V}$ shift in heartbeat's voltage there is a different systolic pressure and diastolic pressure. The values would need to be calibrated for each user as the thickness and skin tone of the finger would be relative to the person.

In order to calibrate the device for each person, it would require the mean heart rate. In this case in Fig. 8.a) the value would be at $3.68 \mathrm{~V}$ and in Fig. 8 b), the value would be at $3.72 \mathrm{~V}$. The mean value will be relative to the blood pressures' mean value of the midpoint. If the person in Fig. 8 a) heart beat had a blood pressure, at the time, of $120 / 80$, we can map out the relative pressures by using the formula [35]:

$$
Y=\left(\left(\frac{(X-A)}{(B-A)}\right) \times(D-C)+C\right)
$$

where $\mathrm{Y}$ is the blood pressure, $\mathrm{X}$ is the voltage value, $\mathrm{A}$ is the minimum value of the heartbeat, $\mathrm{B}$ is the maximum heartbeat value, $\mathrm{D}$ is the minimum DBP and $\mathrm{C}$ is the maximum SBP. For example, using Fig. 8 a), we can find the blood pressure when the voltage is at the mid-point of $3.68 \mathrm{~V}$ : 


$$
Y=\left(\left(\frac{(3.68-3.57)}{(3.8-3.57)}\right) \times(120-80)+80\right)
$$

This gives a pressure of $\sim 100 \mathrm{mmHg}$. We can use the same equation to predict the blood pressure in Fig. 8 b) by replacing $X$ 's values with the maximum and minimum voltage to obtain the pressure of $128 \mathrm{mmHg}$, when the voltage is at $3.85 \mathrm{~V}$ and $87 \mathrm{mmHg}$ and when the voltage is at a low of $3.61 \mathrm{~V}$, leading to an overall blood pressure of $128 / 87$.

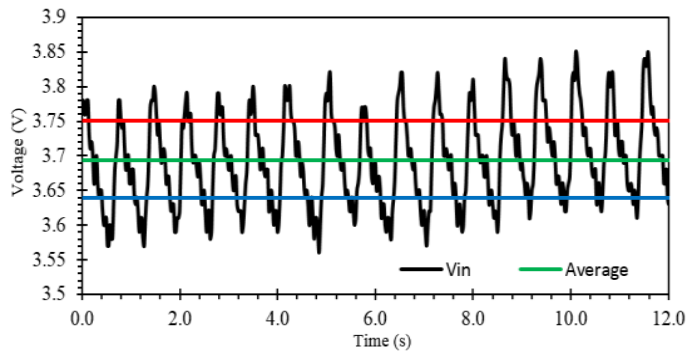

Fig. 9. Heart rate with average, average high and average low.

Fig. 9 shows the graph of the continuous heart rate waveform obtained from the proposed heart rate monitor. It shows about 17 heart beats in 12 seconds, leading to a heart rate of 85BPM. As the average heart rate is measured between 60 and 100 beats per minute and the average blood pressure is $\sim 120 / 80$, it is safe to predict that this person's blood pressure would be $120 / 80$. By taking the average reading of the numbers above the average line (red line $(\mathrm{AvH})$ ) and taking the average reading of the numbers below the average line (blue line (AvL)), we can insert them into the previously stated formula to predict the blood pressure at that moment. From the above expressions and graph reading, the developed model can be expressed as:

$$
B P=\left(\left(\frac{(V i n-A v L)}{A v H-A v L}\right) \times(S B P-D B P)+D B P\right)
$$

where $\mathrm{Y}$ has become the sampled Blood pressure (BP), X has become $\operatorname{Vin}$ (the respective voltage reading from the analogue input), A has become $A v L$ (Average low), B has become $A v H$ (average high), D has become $S B P$ (known systolic blood pressure) and $\mathrm{C}$ has become $D B P$ (known diastolic blood pressure). The sample BP is recorded every $200 \mathrm{~ms}$ and can then be used in a smoothing average formula to view a continuous rate.

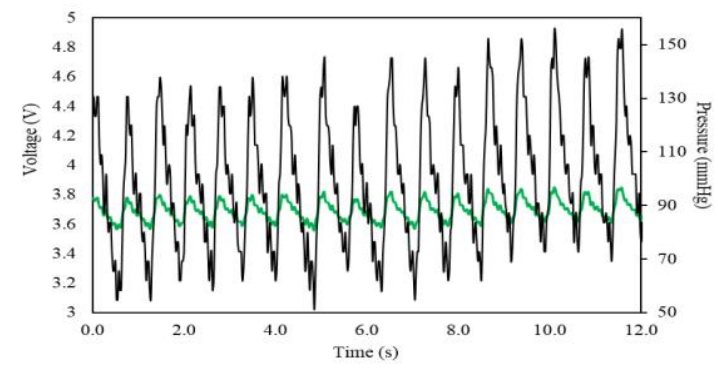

Fig. 10. Correlation between heart rate (green) and blood pressure (black), the heart rate is 88BPM.

The above Figure 10 shows the correlation between the heart rate and blood pressure. It shows that the blood pressure is directly proportional to the heart rate.

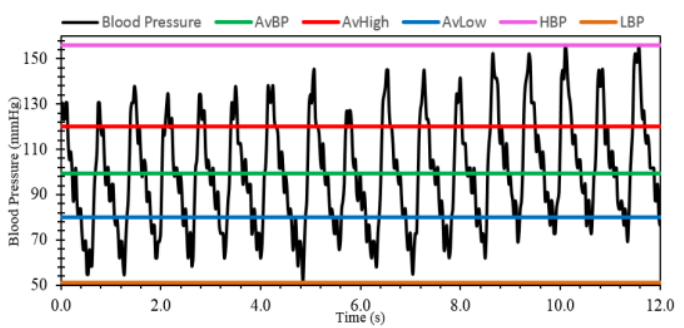

Fig. 11. Predicted average blood pressure calculated using the calibration

Fig. 11 shows the blood pressure's change against time. It ranges from 50 (orange line) to 150 (pink line). The average blood pressure throughout the 12 seconds was at $99.5 \mathrm{mmHg}$ (green line). By taking the average blood pressure above $99.5 \mathrm{mmHg}$, we find the average high to be at $120 \mathrm{mmHg}$ (red line) and by taking the average low of the pressure below the average line, we can obtain an average blood pressure of $80 \mathrm{mmHg}$ (blue line). As Diastole occurs for about two thirds of a heartbeat and systolic for the remaining one third, the equation for Mean Arterial Pressure (MAP) can be expressed as follows [36].

$$
M A P=\left(\frac{((2 \times D P)+S P)}{3}\right)
$$

Given that we can assume systolic and diastolic blood pressure from the heart rate, it is also possible to work out the MAP. Fig. 12 shows blood pressure as a function of time in seconds, the MAP in this Fig. 12 is $93.3 \mathrm{mmHg}$.

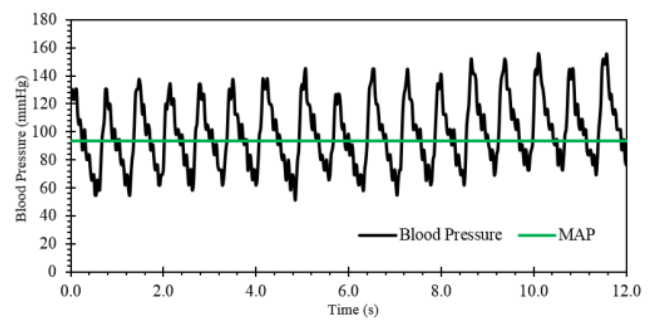

Fig. 12. A graph of MAP of $93.3 \%$ with blood pressure calculated using the calibration.

To further test the theoretical model, another heart rate was conducted with the known blood pressure. The blood pressure during this experiment was 133/72 and it was measured using in-house the Nonin 2120 device [30]. Figure 13 illustrates the heart rate obtained by the heart rate monitor. The heart rate is at 102BPM. 


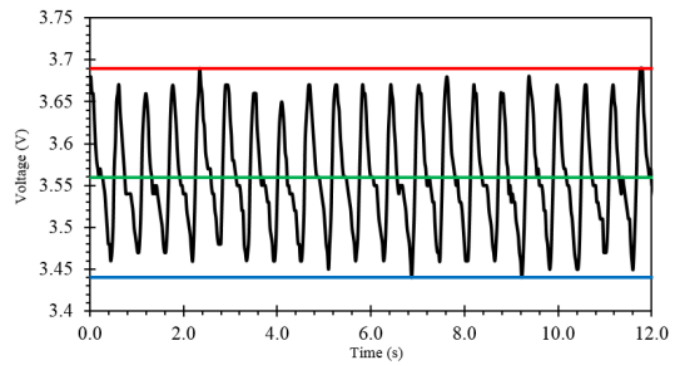

Fig. 13. Heart rate graph with a heart rate of 102BPM and with a blood pressure of $133 / 72$, calculated using the calibration.

The average of the heart rate was taken (green line) along with the maximum (red line) and minimum (blue line). After finding the average high $(3.62 \mathrm{~V})$ and average low $(3.51 \mathrm{~V})$ of the values, it is possible to insert the values into the developed formula (6). Where $A v L$ is $3.51, A v H$ is $3.62, S B P$ is 133 and $D B P$ is 72 . As can be observed the measurement predicted that the average high (systolic blood pressure) was $133 \mathrm{mmHg}$ and the average low (diastolic blood pressure) was at $72 \mathrm{mmHg}$. The blood pressure calibration is illustrated in Fig. 14.

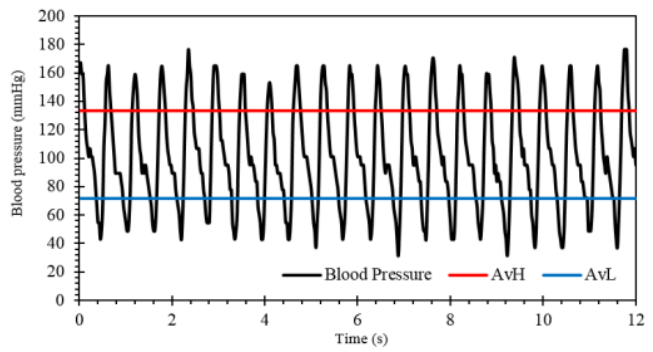

Fig. 14. The blood pressure's calculated using the calibration.

Now the calibration for the blood pressure has been applied, we can predict the blood pressure with a different heart rate, as shown in Figure 15.

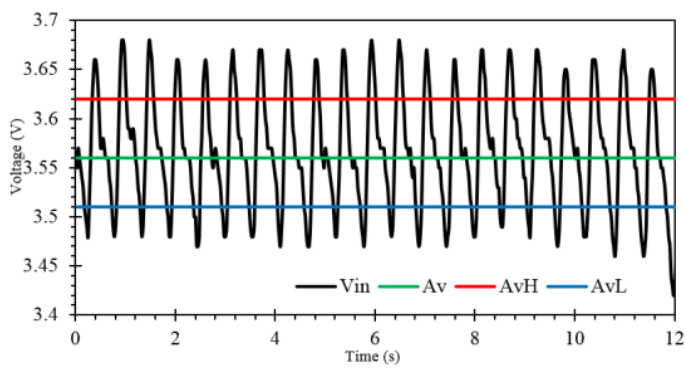

Fig. 15. Heart rate of 105BPM calculated using the calibration.

The values were added to the calibrated formula (7) which predicted a blood pressure of $133.5 / 77$. The real value of the blood pressure was $137 / 77$ (measured by the Nonin 2120 device), showing a $97.4 \%$ accuracy when compared to the benchmark blood pressure monitor. The obtained results of MAP is $95.9 \mathrm{mmHg}$ which is only $1.45 \%$ away from the real MAP value of $97.3 \mathrm{mmHg}$.

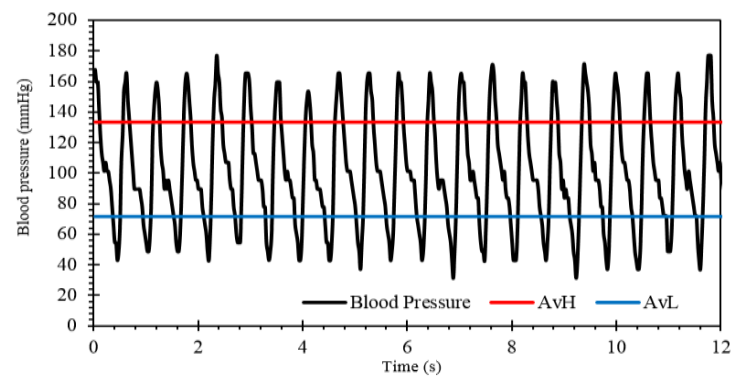

Fig. 16. The heartrate's values used in the model (formula) which predicted the blood pressure of $137 / 77$. calculated using the calibration

Another experiment was carried out for heart rate 95BPM which predicted the blood pressure of 147.5/77, shown in Figure 17. The real blood pressure value measured by the Nonin 2120 was as $160 / 86$, giving a benchmark MAP of $110.7 \mathrm{mmHg}$ and a predicted MAP of $100.5 \mathrm{mmHg}$. Although there is a larger error of $9.2 \%$ within MAP, $7.8 \%$ error within systolic prediction and a $10.5 \%$ error with diastolic pressure prediction, this graph can still represent that there is an apparent correlation between the blood pressure and the heart rate resistance values. The heart rate has not needed to increase dramatically to also produce a high blood pressure. The heart rate is at an average level but the systolic blood pressure is higher than it should be. Although the PPG methodologies have shown some correlation between the overall systolic and diastolic pressures, there could be other variables that may have not been taken into consideration. The blood pressure value does not affect the heart rate and it can be calculated independent of the heart rate.

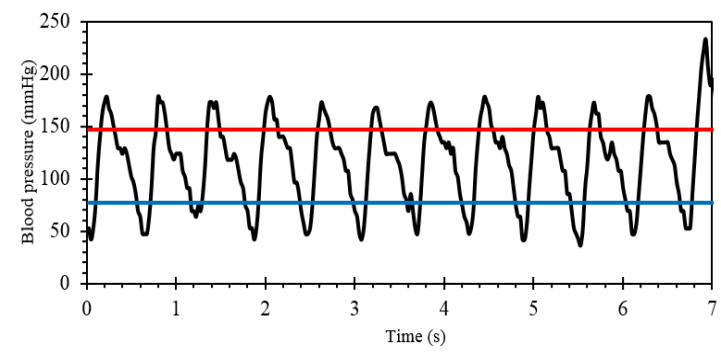

Fig. 17. A graph showing heart rate at 95BPM with the blood pressure of $147.5 / 77$.

Pearson's product moment correlation coefficient can be used to obtain the linearity of the average voltage and the blood pressure [37]. By taking the average voltage of the heart rate monitor after five hundred samples and recording the blood pressure on a bench mark device, we can compare the correlation. The resulting value $r$ will produce a value between -1 and 1 . If the $r$ is equal to 1 , the two variables show strong linear correlation and if the value is close to -1 , the values can be considered highly negatively correlation. A value of 0 shows no correlation. Considering that the systolic and diastolic values are difficult to compare with one variable (the average value), we can compare the average voltage with the MAP (as can be seen in equation (7)).

By taking the $\mathrm{Y}$ values (Average voltage of 500 samples $(12.5$ 
seconds recording the values)) and the $\mathrm{X}$ values (MAP), we can insert them into the formula: $r=s_{x y} / \sqrt{s_{x x} s_{y y}}$ by using the following equations:

$$
\begin{gathered}
S_{x y}=\sum x y-\frac{\sum x \sum y}{n} ; \quad S_{x x}=\sum x^{2}-\frac{\left(\sum x^{2}\right)}{n} \\
S_{y y}=\sum y-\frac{\left(\sum y^{2}\right)}{n}
\end{gathered}
$$

where $\Sigma x$ is the sum of all of the MAPs, $\Sigma y$ is the sum of all of the average voltage values, $\Sigma x^{2}$ is the sum of all of the MAP values squared, $\Sigma y^{2}$ is the sum of all of the average voltage values squared, $\Sigma x y$ is the sum of all of the MAP values multiplied by the average voltage values and $n$ is the total number of variables. Table. 1 below shows the values of $X, Y$, $X 2, Y 2$, and $X Y$ values used for the formulas and Table. 2 shows the sum products; $\Sigma x, \Sigma y, \Sigma x^{2}, \Sigma y^{2}, \Sigma x y$ and $n$.

\begin{tabular}{|c|c|c|c|c|}
\hline MAP & AvVal & $\mathrm{X}^{2}$ & $\mathrm{Y}^{2}$ & $\mathrm{XY}$ \\
\hline 110.333333 & 3.55511022 & 12173.444 & 12.6388087 & 392.24716 \\
\hline 110.666667 & 3.570320641 & 12247.111 & 12.7471895 & 395.11548 \\
\hline 111 & 3.565751503 & 12321 & 12.7145838 & 395.79842 \\
\hline 79 & 3.448416834 & 6241 & 11.8915787 & 272.42493 \\
\hline 81.3333333 & 3.438557114 & 6615.1111 & 11.823675 & 279.66931 \\
\hline 82.3333333 & 3.444749499 & 6778.7778 & 11.8662991 & 283.61771 \\
\hline 88 & 3.448857715 & 7744 & 11.8946195 & 303.49948 \\
\hline 91.6666667 & 3.440420842 & 8402.7778 & 11.8364956 & 315.37191 \\
\hline 102.333333 & 3.509579158 & 10472.111 & 12.3171459 & 359.14693 \\
\hline 102 & 3.467755511 & 10404 & 12.0253283 & 353.71106 \\
\hline 92 & 3.501202405 & 8464 & 12.2584183 & 322.11062 \\
\hline 92.3333333 & 3.444629259 & 8525.4444 & 11.8654707 & 318.0541 \\
\hline 93 & 3.434448898 & 8649 & 11.7954392 & 319.40375 \\
\hline 97 & 3.518456914 & 9409 & 12.3795391 & 341.29032 \\
\hline
\end{tabular}

Table.1. $\mathrm{X}$ and $\mathrm{Y}$ values used to calculate the product moment correlation coefficient

\begin{tabular}{|c|c|c|c|c|c|}
\hline$\Sigma \mathrm{X}$ & $\Sigma \mathrm{Y}$ & $\Sigma \mathrm{X}^{2}$ & $\Sigma \mathrm{Y}^{2}$ & $\Sigma \mathrm{XY}$ & $\mathrm{N}$ \\
\hline 1333 & 48.7883 & 128447 & 170.055 & 4651.46 & 14 \\
\hline
\end{tabular}

Table.2. The sum products and n used for the formulas.

The results proved $S_{x y}$ to be $6.12, S_{x x}$ to be 1526 and $S_{y y}$ to be 0.0336 . The calculated $r$ value is 0.86 . This shows that there is strong correlation between the change in average voltage and the change in the MAP.

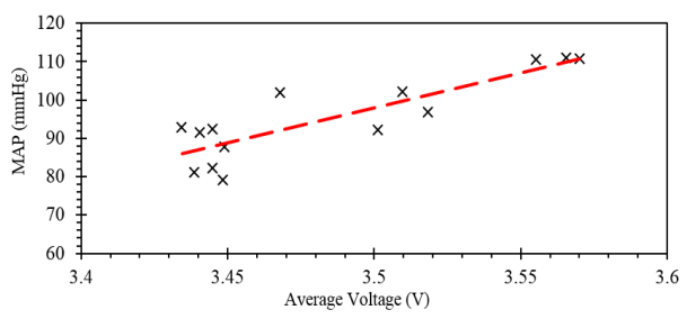

Fig. 18. A graph of average voltage and MAP.
Figure 18 shows the mean arterial pressures ranging from 79 to $111 \mathrm{mmHg}$ against the average voltages ranging from $3.43 \mathrm{~V}$ to 3.57V. Considering the Arduino microcontroller can only take values to two decimal places, correlation can be seen with the values. If the voltage could be measured over a smaller scale, the values would be more spread apart.

In this method by using obtained results in Fig. 18, and equation (6), $\mathrm{Y}=\mathrm{MX}+\mathrm{C}$ formula can be used to predict a mean arterial pressure with voltages, where $\mathrm{Y}$ is the average voltage, $\mathrm{X}$ is the MAP, $\mathrm{M}$ is the gradient (187.09) and $\mathrm{C}$ is the $\mathrm{Y}$ intercept (556.41). The formula is now $\mathrm{Y}=187.09 \mathrm{X}-556.41$. Fig. 19 illustrates the real values calculated (red line) and the predicted values from the equation. An average voltage of 3.40 will predict a MAP of $79.70 \mathrm{mmHg}$, an average voltage of $3.50 \mathrm{~V}$ will predict a MAP of $98.41 \mathrm{mmHg}$ and a voltage of 3.60 will predict a MAP of 117.1. This shows that for every $0.1 \mathrm{~V}$ change in average voltage, $18.7 \mathrm{mmHg}$ change in MAP can be seen. This could be the reason why Fig. 18's clustering results differ from $79 \mathrm{mmHg}$ to $90 \mathrm{mmHg}$ with only a $0.02 \mathrm{~V}$ range. Sample four showed an error of $12 \%$ which is quite large however the rest of the results were never more than $7 \%$ off. As a preliminary experiment, the obtained results clearly demonstrated correlation between the voltages received from the heart rate monitor and the real benchmark blood pressure.

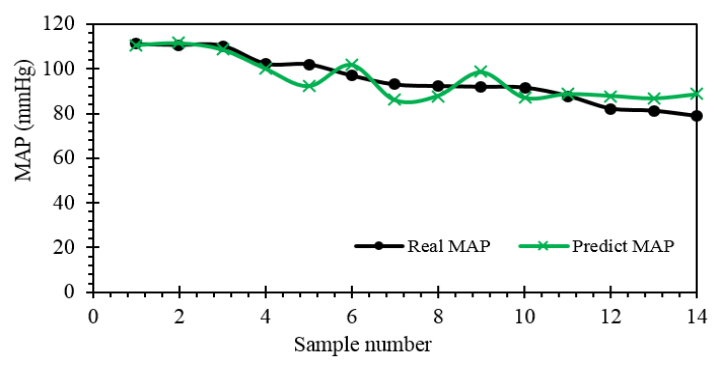

Fig. 19. Real mean arterial pressure values (black line) against the predicted values (green line).

Since we can predict the MAP, we can try to predict the systolic pressure (SP) and diastolic pressure (DP) using the MAP's results. By dividing each value's real systolic pressure by the real MAP, we get a value between 1.40 and 1.46. This value can be averaged out to become 1.4307 (SPk). The same can be carried out by dividing the real diastolic value by the MAP to gain an average of $0.7846(\mathrm{DPk})$. When the averaged $\mathrm{SPk}$ value is multiplied by the predicted MAP, we gain a predicted SP value, and when the DPk value is multiplied by the MAP, we gain a predicted DP value. An experiment, ran 14 times, took the MAP of a participant while the heart rate ringed device was also recording. The original calibration numbers were reused and the resulting values were placed into the new formula. Table 3 shows the Real MAP values, predicted MAP values and the accuracy off the predicted values with the real MAP values. 


\begin{tabular}{|c|c|c|c|}
\hline $\mathrm{n}$ & Real MAP & Predict Map & MAP $\%$ \\
\hline 1 & 110.33 & 108.72 & 1.47 \\
\hline 2 & 110.67 & 111.56 & 0.81 \\
\hline 3 & 111 & 110.71 & 0.26 \\
\hline 4 & 79 & 88.75 & 12.35 \\
\hline 5 & 81.33 & 86.91 & 6.86 \\
\hline 6 & 82.33 & 88.07 & 6.97 \\
\hline 7 & 88 & 88.84 & 0.95 \\
\hline 8 & 91.67 & 87.26 & 4.81 \\
\hline 9 & 102.33 & 100.20 & 2.09 \\
\hline 10 & 102 & 92.37 & 9.44 \\
\hline 11 & 92 & 98.63 & 7.21 \\
\hline 12 & 92.33 & 88.05 & 4.64 \\
\hline 13 & 93 & 86.14 & 7.38 \\
\hline 14 & 97 & 101.86 & 5.01 \\
\hline & & & $5.02 \%$ \\
\hline
\end{tabular}

Table 3. A table of the Real MAP (mmHg) values with the Predicted Values (mmHg) and the errors in the values (\%).

Fig. 20. a) illustrates the real SP values with the predicted values, it is clear from this figure that the values are very close to the real SP values. The red markers show that as the real SP values decrease, so do the predicted values. The accuracy testing of the predicted values to the real values revealed an overall accuracy of $\pm 5 \%$ with the least accurate value being $12 \%$ off its true value (14th sample) and the most accurate being $0.24 \%$ off the real value. The diastolic values could also be predicted to $\pm 5.1 \%$ accuracy of real value, as shown in Fig. 20. b) with very similar correlation.
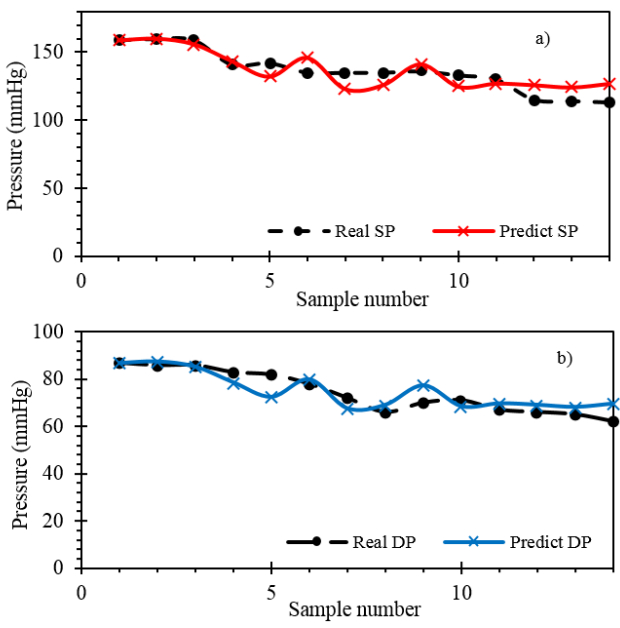

Fig. 20. a) Real systolic pressures (black short dashed line) with the predicted SP pressures (red markers). b) Real diastolic pressures (black long dashed line) with the predicted DP pressures (blue markers).

Figure 21 shows the overall accuracy of the MAP, SP and DP real, and predicted values. The black dotted line shows the real SP values, the black line is the real MAP values, the black dashed line is the real DP values, the red markers are the predicted SP values, green markers are the predicted MAP values and the blue markers are the predicted DP values. The values are predicted with aid of the original participant's calibration to set a base value.

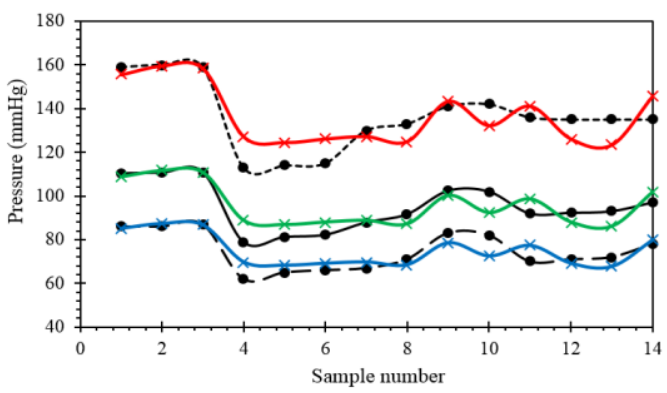

Fig. 21. A combined graph of all of the real pressures (Systolic (black short dashed line), mean arterial pressure (black line) and diastolic pressures (black long dashed line)) with the predicted values (systolic (red), MAP (green) and diastolic (blue) showing the correlation between all values.

Figure 22 a) shows an image of the experiment set up with the blood pressure blocking the left brachial artery. The heart rate device is on the index finger of the right hand sending the changes in voltages to the Arduino's serial monitor for later analysis. Figure $22 \mathrm{~b}$ illustrates the proposed schematic diagram of the system predicting the MAP, SP and DP.

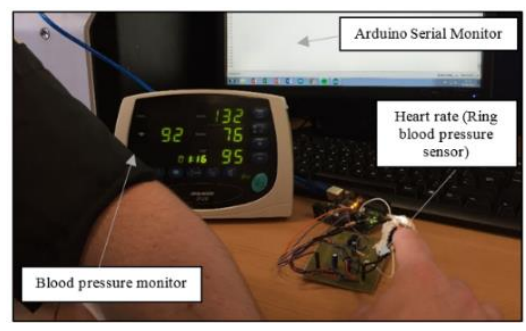

Fig. 22 a). Experiment set up of the measurement blood and the ring sensor measuring the blood pressure.

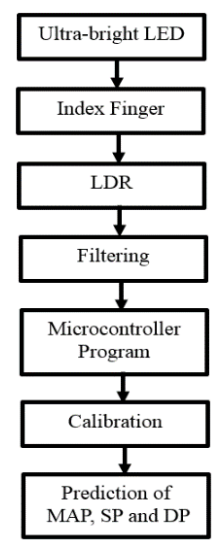

Fig. 22.b Schematic diagram of the system from the LED input to the prediction of the MAP, SP and DP.

It is clear to see that there is a correlation between the average 
heart rate and the corresponding real time blood pressure. Our experiment demonstrates that as there is more blood flowing through the finger, there is a higher voltage received at the heart rate monitor. The higher the voltage received, the higher the corresponding blood pressure of the user. Figure 23 shows a graph of four participants' average voltages taken throughout the day against the real MAP. It is clear to see that there is correlation strong between the voltage and the blood pressure. Each participant (PPT) was of different race to show that the amplitude of the average voltage will change each person's calibration with tone of the skin and thickness of the finger. The voltages show that as the voltages increase, the MAP also increases. A Pearson's product moment correlation coefficient was taken for each participant. The first participant (red line (MAP) and red dashed line (Vin)) has a correlation of 0.88 . The second participant (green line (MAP) and green dashed line (Vin)) has a correlation of 0.924 . The third participant (blue line (MAP) and blue dashed line (Vin)) has a correlation of 0.78 and the fourth participant (purple line (MAP) and purple dashed line (Vin)) has a correlation of 0.90 . The overall average correlation of the participants is 0.87 which suggests strong positive correlation. The values can be found below in table 4 .

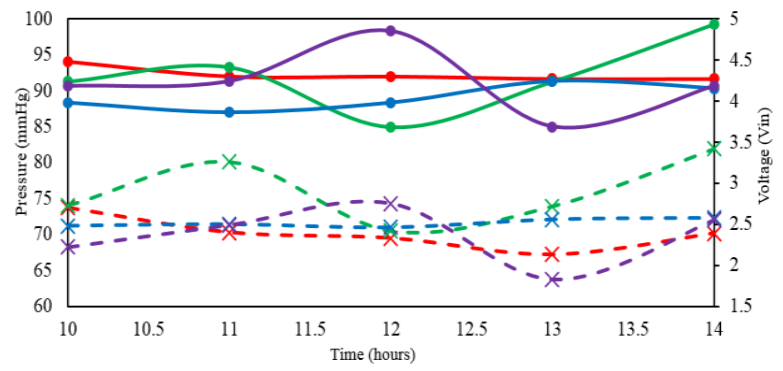

Fig. 23. A colour coded graph of four different participants' real MAP against the average voltage.

\begin{tabular}{|c|c|c|c|c|c|c|c|c|}
\hline Time & MAP1 & Vin1 & MAP2 & Vin2 & MAP3 & Vin3 & MAP4 & Vin4 \\
\hline 10:00 & 94 & 2.7 & 91.333 & 2.73 & 88.333 & 2.48 & 90.6667 & 2.22 \\
\hline $11: 00$ & 92 & 2.4 & 93.333 & 3.26 & 87 & 2.5 & 91.333 & 2.49 \\
\hline $12: 00$ & 92 & 2.33 & 85 & 2.42 & 88.333 & 2.46 & 98.333 & 2.75 \\
\hline $13: 00$ & 91.6667 & 2.13 & 91.3333 & 2.72 & 91.333 & 2.56 & 85 & 1.83 \\
\hline $14: 00$ & 91.6667 & 2.39 & 99.333 & 3.42 & 90.33 & 2.58 & 90.6667 & 2.55 \\
\hline
\end{tabular}

Table 4. A table showing four participants' real mean arterial pressure (MAP) values with the average voltage (Vin) for four participants measured from 10:00am to 14:00pm.

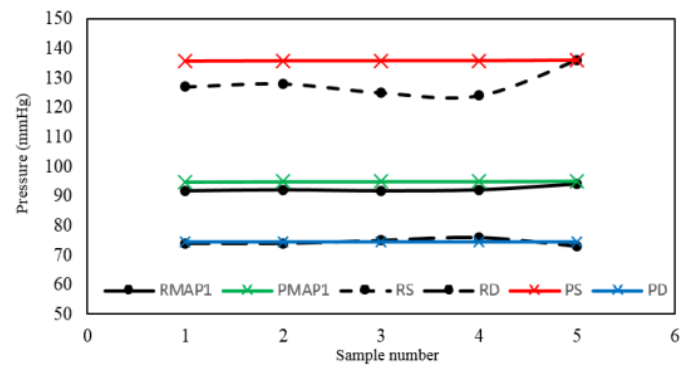

Fig. 24. A graph of the real MAP of the first participant's real pressures (real systolic (RS, black small dashed line), real MAP
(RMAP1, black line) and diastolic (RD, black large dashed line)) with the predicted pressures (predicted systolic (PS, red line), predicted MAP (PMAP1, green line) and predicted diastolic (PD, blue line)).

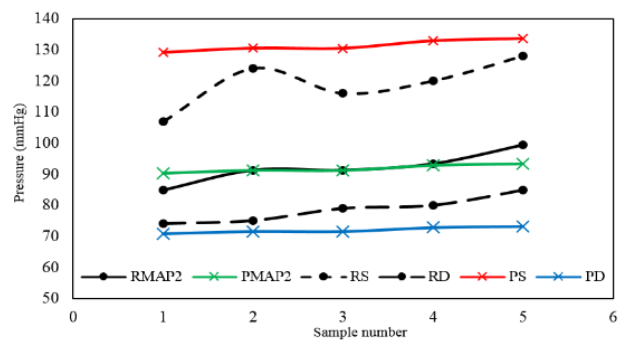

Fig. 25. A graph of the real MAP of the second participant's real pressures (real systolic (RS, black small dashed line), real MAP (RMAP2, black line) and diastolic (RD, black large dashed line)) with the predicted pressures (predicted systolic (PS, red line), predicted MAP (PMAP2, green line) and predicted diastolic (PD, blue line)).

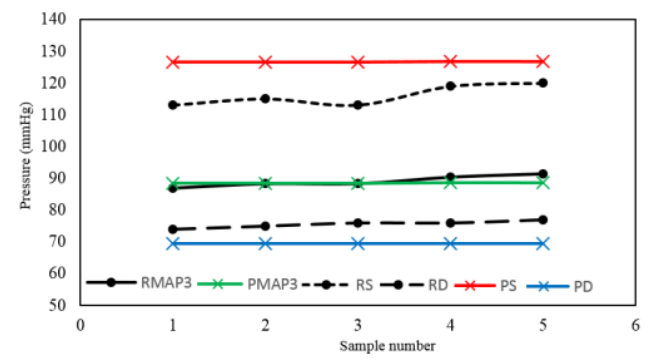

Fig. 26. A graph of the real MAP of the third participant's real pressures (real systolic (RS, black small dashed line), real MAP (RMAP3, black line) and diastolic (RD, black large dashed line)) with the predicted pressures (predicted systolic (PS, red line), predicted MAP (PMAP3, green line) and predicted diastolic (PD, blue line)).

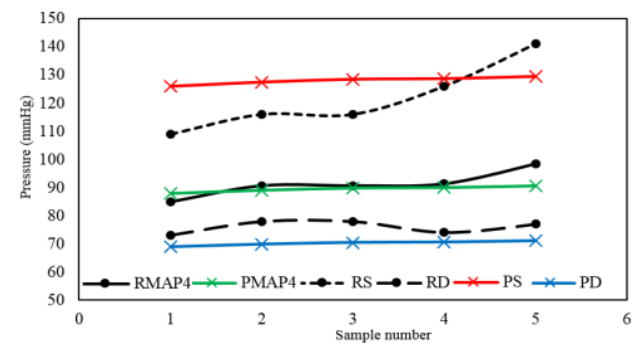

Fig. 27. A graph of the real MAP of the fourth participant's real pressures (real systolic (RS, black small dashed line), real MAP (RMAP4, black line) and diastolic (RD, black large dashed line)) with the predicted pressures (predicted systolic (PS, red line), predicted MAP (PMAP4, green line) and predicted diastolic (PD, blue line)).

Figures 24-27 show the four participant's real pressures against the predicted pressures. The first and third participants claimed to have not eaten anything during the course of the day. This 
would explain why the values remain at a steak level. All of the systolic and diastolic pressures seem to be slightly off. Although they do resemble over $90 \%$ accuracy of the predicted pressures. The same SPk and DPk values were used to calibrate the systolic and diastolic pressures and may need more results to gain a more accurate value. All of the predicted mean arterial pressures were over $93 \%$ accurate which suggests that there is correlation between the average voltage of the heart rate monitor and the mean arterial pressure. In regards to the mean arterial pressure, PPT 1 has a real MAP range of $91.667-94 \mathrm{mmHg}$ and a predicted range from $94.8-95.12 \mathrm{mmHg}$, an overall average accuracy of $97 \%$ throughout the five hours. PPT 2 has a real MAP range of $85-99.33 \mathrm{mmHg}$ and a predicted range from $90.29-93.36 \mathrm{mmHg}$, an overall average accuracy of $99 \%$ throughout the five hours. PPT 3 has a real MAP range of $87-91.33 \mathrm{mmHg}$ and a predicted range from $88.53-88.60 \mathrm{mmHg}$, an overall average accuracy of $99 \%$ throughout the five hours. PPT 4 has a real MAP range of $85-98.33 \mathrm{mmHg}$ and a predicted range from $88.0-90.51 \mathrm{mmHg}$, an overall average accuracy of $98 \%$ throughout the five hours. It is clear to see that the five tests did not produce results with more diverse values. If the ranges of the participants were longer, the calibration of the participants would have produced more accurate results like Figure 21. Figures 24-27 use the same calibration techniques as Figure 21 and uses the changes in the average voltage to view the change in blood pressure.

Figure 28 illustrates the measurements of one participant blood pressure which is carried out from 10:30h till 14:30h. It shows the blood pressure changing against the predicted values. After further calibration, the average MAP of the four hours was at $98.92 \mathrm{mmHg}$ and the average predicted MAP over the four hours was at $92.8 \mathrm{mmHg}(93.8 \%$ accurate). The average real systolic pressure was at $144.25 \mathrm{mmHg}$ and the predicted average systolic pressure was at $132.77 \mathrm{mmHg}(92 \%$ accurate). The average real diastolic pressure was at $76.25 \mathrm{mmHg}$ and the predicted diastolic pressure was at $72.7 \mathrm{mmHg}(95.5 \%$ accurate $)$. The participant had lunch at 13:00h and it is clear to see from the graph, an increase in real and predicted blood pressure (at 13:30) and restores back to near the average values at 14:00h. Our experiment proves that continuous monitoring of the blood pressure with this device throughout the day and night is possible though miniaturisation by incorporating smaller components (i.e. 0402 for the Band Pass Filter) would be required.

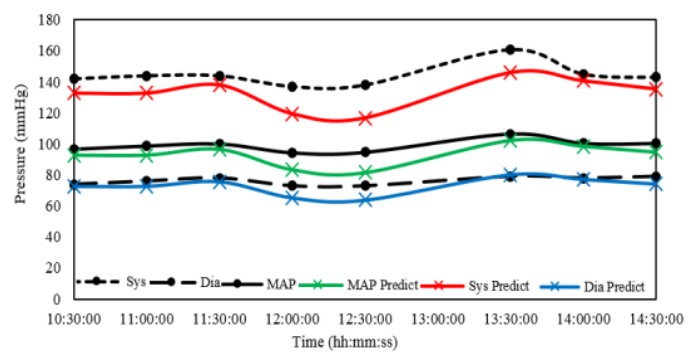

Fig. 28. Graph of real MAP (black line), systolic (black short dash) and diastolic (black long dash) pressures with the predicted; MAP (green line), systolic (red line) and diastolic

(blue line) against time from 10:30am to $14: 30 \mathrm{pm}$.
The average real heart rate throughout the experiment ranged from 74-108BPM and the predicted heart rate ranged from 77.7115.2BPM. The heart rate accuracy throughout the experiment was at $104 \%$. This shows that slight calibration within the programming is required though it is still highly accurate.

\section{CONCLUSION}

In this research paper we have proposed and demonstrated a novel continuous monitoring of the blood pressure using a ring as a simple and low cost heart rate sensor. We have demonstrated that it could be possible to obtain a predicted blood pressure using that proposed heart rate device. We show that when more blood is flowing through the finger (more blood in the finger), less light is received by the LDR which created a larger resistance. As there is a larger resistance, there is a higher blood pressure. The mean arterial blood pressure can be predicted with aid of the photoplethysmography, and systolic and diastolic pressures can be predicted with use of the MAP to $5 \%$ of their real values. This method uses a different technique of measuring blood pressure compared with the current devices that still use the cuff method like the Nonin 2120 benchmark blood pressure device [30]. Not using the cuff method and having a continuous blood pressure measurement throughout the day will eliminate any issues patients may have when having their blood pressure taken. This method will enable continuous monitoring of the blood pressure where the medical examiners will be able to view how a patient's blood pressure changes throughout the day and gain a true value of the average blood pressure as food, caffeine and other variables can have significant and measurable effects on the blood pressure. The stress of having spot blood pressure readings taken in itself can affect the reading. This novel device is extremely affordable using basic filtering and amplification techniques. In this paper we show that there is a strong linear correlation between the amplitude of the voltage received and the blood pressure, enabling to build the continuous blood pressure sensor. The proposed blood pressure device is tested and benchmarked against Nonin 2120 [30] for four participants for a continuous period of four hours. The demonstrated device accuracy between average real systolic pressure and the predicted average systolic pressure was $92 \%$. The demonstrated device accuracy for the average real diastolic pressure and the predicted diastolic pressure is $95.5 \%$. The demonstrated accuracy between real average MAP and the average predicted MAP, is $93.8 \%$.

\section{ACKNOWLEDGMENT}

The authors would like to thank the participants for their help with testing and result and Mitchell Cohen for his contribution in reading and improving the manuscript.

\section{REFERENCES AND FOOTNOTES}

[1] C. Black "Cardiovascular diseases" World Health Organization Available at: http://www.who.int/cardiovascular_diseases/en/ (2016)

[2] "The Future" World Health Organization Available at: 
http://www.who.int/cardiovascular_diseases/en/cvd_atlas_ 25_future.pdf?ua=1 (2016)

[3] "The history of our heart attack research" British Heart Foundation Available at: https://www.bhf.org.uk/lifesaving-research/heart-attack-history (2016)

[4] P. B. Adamson, W. T. Abraham, M. Aaron, J. M Jr Aranda, R. C. Bourge, A. Smith, L. W. Stevenson, J. G. Bauman and J. S. Yadav. "Champion trial rationale and design: the long-term safety and clinical efficacy of a wireless pulmonary artery pressure monitoring system. " $J$ Card Fail. vol.. 17, pp. 3-10, 2011.

[5] Y. L. Zheng, XR. Ding, Poon, C.C.Y, Lo, B.P.L., H. Zhang, XL. Zhou, G.Z. Yang, N. Zhao and YT Zhang, "Unobtrusive Sensing and Wearable Devices for Health Informatics." Biomedical Engineering, IEEE, vol. 61, pp. 538-1554.2014.

[6] W. Nahm and H. Gehring, "Non-invasive in vivo measurement of blood spectrum by time-resolved nearinfrared spectroscopy." Sensors and Actuators B, 29, pp. 174-179. 1995.

[7] M. Rothmaier, B. Selm, S. Spichtig, D. Haensse, and M. Wolf, "Photonic textiles for pulse oximetry", Optics Express, vol. 16, pp. 12973-12985, 2008.

[8] A. F. Martins, D. F. S. Santos, A. Perkusich and H. O. Almeida "Integrating Personal Health Devices in Home Networks", IEEE 11th Consumer Communications and Networking Conference (CCNC), pp. 1- 6, 2014.

[9] S. Jeong, S. Kim, D. Kim, C. Yun and Y. Kim, “A Personalized Healthcare System for Chronic Disease Care in Home-Hospital Cloud Environments", International Conference on ICT Convergence (ICTC), pp. 371 - 376, 2013.

[10] J. S. Kim, B. O. Kim, and K. S. Park, "Development of HIHM (Home Integrated Health Monitor) for Ubiquitous Home Healthcare" 29th Annual International Conference of the IEEE Engineering in Medicine and Biology Society, $363-365,2007$.

[11] F. D. Anderson "Taking Blood Pressure Correctly", Steeles, Available at: http://www.steeles.com/catalog/takingBP.html

[12] "Sphygmomanometer" [Online] Available at: http://www.practicalclinicalskills.com/sphygmomanomete r.aspx 2016.

[13] W. Li, X. Wang, L. Lu, H. Li, "Discrepancy of blood pressure between the brachial artery and radial artery" World J Emerg Med; vol. 4, pp. 294-297, 2013.

[14] "Blood pressure chart" [Online] Available at: http://www.bloodpressureuk.org/BloodPressureandyou/Th ebasics/Bloodpressurechart, 2008.

[15] W. Tao, T. Liu, R. Zheng and H. Feng, "Gait analysis using wearable sensors". Sensors (Basel), vol. 4, pp. 2255-83, 2012.

[16] O. Olguin, P. Gloor, \& A. Pentland, "Wearable Sensors for Pervasive Healthcare Management." Principles and applications," Proceedings of the IEEE, vol. 66, pp. 1-4, 2009.

[17] G. Appelboom, E. Camacho, M. E. Abraham, S. S.
Bruce, E. L. P. Dumont, B. E. Zacharia, R. D’Amico, J. Slomian, J. Y. Redinster, O. Bruyère and E. S. Collony "Smart wearable body sensors for patient self-assessment and monitoring." Arch. Public Health, 72, p. 28, 2014.

[18] N. Luo, J. Ding, N. Zhao, B. H. K. Leung \& C. C. Y. Poon, "Mobile health: Design of flexible and stretchable electrophysiological sensors for wearable healthcare systems." 11th International Conference on Wearable and Implantable Body Sensor Networks, BSN 2014, pp. 8791. doi:10.1109/BSN.2014.25

[19] S. Yao, \& Y. Zhu, "Wearable multifunctional sensors using printed stretchable conductors made of silver nanowires." Nanoscale, vol. 6, p. 2345, 2014.

[20] D. Vilela, A. Romeo \& S. Sánchez, "Flexible sensors for biomedical technology." Lab Chip (2016). doi:10.1039/C5LC90136G

[21] M. Caldara, C. Colleoni, E. Guido, G. Rosace, V. Re and A. Vitali, "A wearable sensor platform to monitor sweat $\mathrm{pH}$ and skin temperature." IEEE International Conference on Body Sensor Networks, BSN 2013 (2013). doi:10.1109/BSN.2013.6575465.

[22] A. J. Bandodkar \& J. Wang, "Non-invasive wearable electrochemical sensors: A review." Trends in Biotechnology, 2014, 32, pp. 363-371.

[23] Z. J. V. Cohen, S. Haxha, and A. Aggoun, "Pulse Oximetry Optical Sensor using Oxygen-Bound Haemoglobin" Optics Express, vol. 24, pp. 10115-10131, 2016.

[24] R. V. Kanawade, N. Alhamwi, F. Klämpfl, M. Riemann, C. Knipfer, M. Schmidt, and Florian Stelzle, "Photoplethysmography (PPG) Sensor for Real-time Body Hemodynamics Monitoring - An Efficient, Robust and Simple Approach for Clinical Shock Diagnostics". Optics Express, Biomedial Optics 2014.

[25] L. Florea \& D. Diamond, "Advances in wearable chemical sensor design for monitoring biological fluids." Sensors Actuators B Chem. 2015, 211, pp. 403-418.

[26] F. D. Anderson "Taking Blood Pressure Correctly", Steeles, Available at: http://www.steeles.com/catalog/takingBP.html

[27] "Connected Arm Blood Pressure Monitor" [Online] Available at: http://www.ihealthlabs.eu/en/wireless-bloodpressure-monitors/9-wireless-blood-pressuremonitor.html, 2015.

[28] "Connected Wrist Blood Pressure Monitor" [Online] Available at: http://www.ihealthlabs.eu/en/wireless-bloodpressure-monitors/8-wireless-blood-pressure-wristmonitor.html, 2015.

[29]A. Trafton, "Wearable blood pressure sensor offers 24/7 continuous monitoring" [Online] Available at: http://news.mit.edu/2009/blood-pressure-tt0408 2009.

[30] "2120 Table top Pulse Oximeter w/Non-invasive Blood Pressure" Nonin Medical Inc. Available at: http://www.nonin.com/Avant2120, 2016.

[31] M. Islam, F. Hasan, A. Mitul and M. Ahmad, "Development of a Non-invasive Continuous Blood Pressure Measurement and Monitoring System" 
International Conference on Informatics, Electronics \& Vision, pp. 1085 - 1090, 2012.

[32] D. C. Zheng and Y. T. Zhang, "A Ring-type Device for the Noninvasive Measurement of Arterial Blood Pressure" International Conference of the IEEE EMBS, pp. 31843187, 2003.

[33] R. Shriram, A. Wakankar, N. Daimiwal and D. Ramdasi "Continuous Cuffless Blood Pressure Monitoring Based on PTT" Department of Instrumentation and Control Cummins College of Engineering for Women, IEEE, pp. 51-55, 2010.

[34] Y. Z. Yoon and G. W. Yoon "Non-constrained Blood Pressure Measurement by Photoplethysmography" Journal of the Optical Society of Korea, 2006, 10, pp. 91-95.

[35] P. A. Webb, "Math - mapping numbers" [Online] Available at: http://stackoverflow.com/questions/345187/mathmapping-numbers, 2008.

[36] L. Bonsall, "Calculating the MAP" [Online] Available at: https://www.nursingcenter.com/NCBlog/December2011/Calculating-the-MAP, 2011.

[37] M. J. Campbell, editor. Swinscow TDV. In: Statistics at square one. Ninth Edition. Copyright BMJ Publishing Group, 1997. 